\title{
Human endogenous retrovirus HERV-K(HML-2) envexpression is not associated with markers of immunosenescence
}

Saara M arttilaa , Tapio Nevalainen ${ }^{a}$, Juulia Jylhäväa,b, Laura Kananena , M arja Jylhäc, Antti Hervonenc, M ikko Hurme $^{\mathrm{a}}$

aFaculty of Medicine and Biosciences, University of Tampere, Tampere, Finland; Postal address: Faculty of M edicine and Life Sciences, FI-33014 University of Tampere, Finland

bPresent address: Department of M edical Epidemiology and Biostatistics, Karolinska Institute, Stockholm, Sweden; Postal address: Department of Medical Epidemiology and Biostatistics, Karolinska Institute, PO Box 281, SE-171 77 Stockholm, Sweden

'Faculty of Social Sciences and Gerontology Research Center, University of Tampere, Tampere, Finland; Postal address: Faculty of Social Sciences, FI-33014 University of Tampere, Finland

Corresponding author:

Dr. Saara M arttila

Faculty of M edicine and Biosciences, University of Tampere

Tampere, FI-33014, Finland

e-mail saara.marttila@staff.uta.fi

TN: tapio.I.nevalainen@staff.uta.fi

JJ: juulia.jylhava@ki.se

LK: laura.kananen@staff.uta.fi

MJ: marja.jylha@uta.fi

AH: antti.hervonen@uta.fi

M H: mikko.a.hurme@uta.fi 


\begin{abstract}
Ageing of the human immune system, or immunosenescence, is characterised by distinct changes in the proportion of the various cell types, e.g., increase of the CD14+ monocytic cells, decrease of CD19+ B lymphocytes, and changes in T cell subpopulations, namely increase of CD4+and CD8+cells which have lost the costimulatory CD28 antigen. Currently, it is believed that the lifelong antigenic burden may be one of the inducers of immunosenescence. Thus far, only one exogenous stimulus, cytomegalovirus infection, has shown to be a major factor in this respect. To find other possible candidates, we evaluated the role of the evolutionary youngest group of human endogenous retroviruses, namely HERV-K(HML-2), on immunosenescence. HERVs exist in the genome as proviruses, but their activation has been detected in several immunopathologic conditions. The expression of HERV-K(HML-2) env was observed to be lower in the peripheral blood mononuclear cells of nonagenarians $(n=61)$ than in those of young controls $(n=37)$. These mRNA levels did not correlate with the age-associated differences in the proportions of CD14+, CD4+CD28- and CD8+CD28- cells, but in the case of CD19+B cells a strong positive correlation was observed in the nonagenarians. Thus, these data suggest that HERVs do not function as antigenic drivers of immunosenescence. On the contrary, expression of HERV-K(HM L-2) env is associated with more youthful levels of B cells.
\end{abstract}

Keywords: human endogenous retrovirus, HERV-K(HM L-2), aging, immunosenescence, B cells 


\section{Introduction}

The ageing-associated changes in the immune system have been extensively characterised during the last few decades (reviewed in Lang et al. 2013, Goronzy et al. 2013). The hallmarks of immunosenescence are the decreased number of naïve T cells and accumulation of late-stage memory T cells. In the B cell compartment, this decline of naïve cells is also detectable. Ageing is also associated with low-level increase in inflammatory mediators, termed inflamm-aging (reviewed in Franceschi et al. 2014). At the cellular level, one important question has yet to be answered: is immunosenescence an intrinsic ageing process or an adaptive response to an individual pathogen exposure? Thus far, only one pathogen, human cytomegalovirus (CM V), seems to fulfill the criteria of an extrinsic antigen. CMV infection takes place usually in early childhood and the virus remains in a latent form in the body. In elderly individuals, the seropositivity rate is $70-100 \%$. The associations between CM V seropositivity and the ageing-associated changes in the various cell types and functions of the immune system have been extensively demonstrated (reviewed in Söderberg-Naucler et al. 2016, Weltevrede et al. 2016). However, ageing is associated with changes in the function and composition of the immune system also in CMV seronegative individuals (Di Benedetto et al., 2015). In addition, with genomewide transcriptomic analysis we have identified ageing-associated changes in the immune system in both CMV seronegative and CMV seropositive individuals and have demonstrated that the identified changes differ between CM V seronegative and seropositive individuals (Kuparinen et al., 2013). These results suggest that $\mathrm{CMV}$ is only partially responsible for the induction of immunosenescence.

In this study, we analyse the role of an additional virus group, i.e., the human endogenous retroviruses (HERVs) in immunosenescence. HERVs are remnants of ancient infections and have remained in the human genome as proviruses, comprising about $8 \%$ of the genome (M agiorkinis et al. 2013). Previous research show that these proviruses, including the evolutionary youngest ones, the HERV-K(HML-2) group members, can be expressed in several pathological conditions, e.g., in autoimmune diseases such as rheumatoid arthritis, multiple sclerosis and systemic lupus erythematosus (as reviewed in Magiorkinis et al. 2013). However, no direct causal mechanism has been demonstrated between HERVs and pathological conditions. For instance, no reports show that HERVs have retrotransposonal capacity, and the possible pathogenic effects are thought to be mediated via viral RNA and protein (reviewed in Suntsova et al., 2015). In addition, HERV sequences in the genome may function as regulatory elements and, thus, modify the expression of nearby genes (Magiorkinis et al. 2013, Volkman \& Stetson 2014). In this study, we investigate whether the HERV-K(HM L-2) RNA could contribute to immunosenescence as shown in exogenous pathogens. 


\section{Materials and Methods}

The study population consist of 61 nonagenarians born in 1920 ( 21 males, 40 females) and 37 young controls (healthy laboratory personnel, aged 19-34 years, 12 males, 25 females), who participated in the Vitality $90+$ study in 2014. The Vitality 90+study is an ongoing, prospective, population-based study that includes both home dwelling and institutionalized individuals who are 90 years of age or older, and who live in the city of Tampere, Finland. The recruitment and characterisation of the participants were performed as has been reported previously (Goebeler et al. 2003). The study subjects were all of Western European descent and had not had previous infections or received any vaccinations in the 30 days prior to the blood sample collection. The study participants provided their written and informed consent to participate in the study. This study was conducted according to the principles expressed in the declaration of Helsinki. The study protocol was approved by the ethics committee of the city of Tampere (1592/403/1996).

Blood samples from nonagenarians were collected by a trained laboratory technician during a home visit and the samples from young controls were collected by a trained laboratory technician in the laboratory facilities. All blood samples were drawn between 8 am and 12 am and collected into EDTA containing tubes. Samples were directly subjected to leucocyte separation on a Ficoll-Paque density gradient (Ficoll-Paque ${ }^{\mathrm{Tm}}$ Premium, cat. no. 17-5442-03, GE Healthcare Bio-Sciences AB, Uppsala, Sweden). The plasma layer was collected and stored at $-70^{\circ} \mathrm{C}$. The PBM C layer was collected and cells used for RNA extraction were suspended in $150 \mu$ l of RNAlater solution (Ambion Inc., Austin, TX, USA). Cells used for FACS analysis were suspended in $1 \mathrm{ml}$ of a freezing solution (5/8 FBS, 2/8 RPM I-160 medium, 1/ 8 DM SO) (FBS cat. no. F7524, Sigma-Aldrich, M 0, USA; RPM I: cat. no. R0883, Sigma-Aldrich, M O, USA; DM SO: cat. no. 1.02931.0500, VWR, Espoo, Finland) and stored in liquid nitrogen.

The anti-CMV IgG titre was measured using a commercial enzyme-linked immunosorbent assay kit (Enzygnost ${ }^{\circledR}$ Anti-CMV/lgG, Siemens Healthcare Diagnostics Products GmbH, Marburg, Germany). Seropositivity for CM V was defined according to the manufacturer's instructions as a plasma anti-CM V IgG titre $\geq 230 \mathrm{U} / \mathrm{ml}$. The plasma C-reactive protein (CRP) concentration was measured using Human CRP Immunoassay (Quantikine ELISA, R\&D Systems, M inneapolis, M N, USA).

The proportions of different leukocyte subtypes were determined using fluorescence-activated cell sorting analysis (FACS; BD FACSCanto II). The results were analysed with FlowJo software (Tree Star Inc., Ashland, OR, USA). The antibodies used were FITC-CD14 (cat. no. 11-0149), PerCP-Cy5.5-CD3 (45-0037), APC-CD28 (170289), PE-CD19 (12-0199) (eBioscience, San Diego, CA, USA), PE-Cy ${ }^{\text {TM }} 7-C D 4$ (cat. no. 557852), and APC-Cy ${ }^{\text {TM } 7-~}$ CD8 (557834) (BD Biosciences). Staining was performed in phosphate-buffered saline (PBS) containing 1\% foetal bovine serum (FBS) after an incubation step with Fc Receptor Binding Inhibitor (cat. no 16-9161, eBioscience) to minimise non-specific staining of the cells. 
RNA was purified using RNeasy mini kit (Qiagen, CA, USA) according to manufacturer's protocol with oncolumn DNA digestion (Qiagen). The concentration and quality of the RNA were assessed with a NanoDrop ND-1000 spectrophotometer (NanoDrop Technologies, Wilmington, DE, USA). 300 ng of RNA was reverse transcribed to cDNA with High-Capacity cDNA Reverse Transcription Kit (Applied Biosystems, CA, USA). The qPCR was performed with PowerUp SYBR Green Master Mix (Applied Biosystems, MA, USA) in 7900HT (Applied Biosystems). Primers (Invitrogen) were used in $500 \mathrm{nM}$ concentration with $6 \mathrm{ng}$ of template per 20 $\mu l$ reaction. Primers used for HERV-K(HML-2) env; forward CTAACCATGTCCCAGTGATG and reverse GGAGACAGACTCATGAGCTTAGAA (Bhardwaj et al., 2014). GUSB was used as an endogenous control; primers forward TGCAGGTGATGGAAGAAGTG and reverse TTGCTCACAAAGGTCACAGG (Beer et al, 2015). The PCR reactions were run as $2^{\prime}$ in $50^{\circ} \mathrm{C}, 2^{\prime}$ in $95^{\circ} \mathrm{C}$ and 40 cycles of $15^{\prime \prime}$ in $95^{\circ} \mathrm{C}, 15^{\prime \prime}$ in $57^{\circ} \mathrm{C}$, and $1^{\prime}$ in $72{ }^{\circ} \mathrm{C}$. All samples were run in triplicate. Expression level is expressed as $\Delta \mathrm{Ct}$ value, calculated as $\mathrm{Ct}(\mathrm{env})$ - $\mathrm{Ct}(\mathrm{GUSB})$. As a higher $\mathrm{Ct}$ value indicates lower expression level, and the expression level of GUSB is higher than that of HERV-K(HM L-2) env, $\Delta \mathrm{Ct}(\mathrm{env})$ is high for low expression levels and vice versa.

\section{Results}

The age-associated differences in the proportions of the various immune cells in our study population were similar to those reported previously in the literature. The proportion of CD14+ monocytic cells, CD4+CD28-, and CD8+CD28- T cells were increased and the proportion of CD19+ B cells were decreased in the nonagenarians as compared to the young controls (Table 1). HERV-K(HM L-2) activity was analysed using the expression of env. This expression was detectable in both nonagenarians and young controls, but the expression was slightly higher in the young controls (Table 1). To analyse the biological significance of HERVK(HM L-2) env expression, the expression levels were correlated with the cell type proportions. In nonagenarians, the expression of HERV-K(HM L-2) env was correlated with the proportion of CD19+B cells, with higher expression level associated with higher proportion of B cells (Table 2). The proportions of other measured cell types were not associated with expression of HERV-K(HML-2) env in nonagenarians. In the young controls HERV-K(HM L-2) envexpression was not associated with the proportions of any measured cell type (Table 2).

To analyse the role of HERV-K(HM L-2) env in inflamm-aging, the expression level was correlated with the plasma level of CRP. However, no association was found in the nonagenarians (Spearman's $\rho=-0.100, p=$ 0.446 ) or in the young controls (Spearman's $\rho=-0.129, p=0.446$ ). The association of expression level of HERVK(HM L-2) env and anti-CMV IgG titer was also analysed, but no association was found among the nonagenarians (Spearman's $\rho=0.012, p=0.926$ ) or in young controls (Spearman's $\rho=-0.017, p=0.918$ ). 


\section{Discussion}

Our data reveal that the expression of HERV-K(HM L-2) env is decreased in nonagenarians as compared to young controls. In addition, the expression of HERV-K(HM L-2) env is not associated with classical cellular markers of immunosenescence. Thus, HERV-K(HML-2) env expression does not contribute to immunosensescence. However, HERV-K(HM L-2) env expression correlated positively with the percentage of the CD19+B cells in nonagenarians.

To the best of our knowledge, age-associated differences in HERV-K(HM L-2) expression have not been reported in the literature previously. However, Balestrieri et al. (2015) analysed the ageing-associated expression differences in the whole HERV-K group. Contrary to our results, they show that children aged under 12 years have lower expression levels of HERV-K, but in adulthood there are no significant changes in HERV-K expression. As HERV-K(HM L-2) is only one of the ten HERV-K HML groups, it appears that different HML groups are expressed independently of each other.

Changes in HERV expression have been reported in various diseases, including various cancers, rheumatoid arthritis, multiple sclerosis and in HIV infected patients. Contrary to ageing, these conditions are associated with increased expression of HERVs (reviewed in Magiorkinis et al., 2013). Also, the difference in HERVK(HM L-2) envexpression between nonagenarians and young controls was modest compared to some of the reported differences in HERV expression between diseased individuals and healthy controls (Freimanis et al., 2010; Morandi et al., 2017).

The observed positive association between HERV-K(HM L-2) envand B cells may be connected to the recent findings in Zeng et al. (2015). They observed that, in mice, B cell receptor crosslinking by T-cell independent antigens activates a signaling cascade, involving NF- $\mathrm{kB}$, that leads to transcription of retroviral RNA and further to $B$ cell activation and differentiation. HERV-K(HML-2) transcription was also shown to be upregulated by B cell receptor signaling in human B cells in vitro (Zeng et al., 2015). Thus, it may be that this positive or immunostimulatory effect of $\mathrm{HERV}-\mathrm{K}(\mathrm{HM} \mathrm{L}-2)$ is related to increased survival or proliferation of $B$ cells in elderly individuals. We have shown that both IgG and IgA serum levels are significantly increased in nonagenarians as compared to those of middle-aged controls (Hurme et al. 2005). It is also possible that HERV-K(HM L-2) envis predominantly expressed in B cells or in some B cell subpopulation.

Previous reports show an association between HERV expression and the inflammatory response, i.e., it is likely that increased production of HERV nucleic acids is able to activate inflammation (reviewed in Volkman \& Stetson, 2014). Although increased inflammation, i.e., inflamm-aging (reviewed in Franceschi et al. 2014) is one of the hallmarks of ageing, our results suggest that HERV-K(HM L-2) envexpression does not contribute to this phenomenon, as env mRNA levels were not associated with percentage of CD14+ cells (Table 2) or levels of CRP. 
Previous report shows that CM V is able to induce expression of HERVs, including HERV-K(HM L-2), in cultured cancerous and normal cell lines as well as monocytes derived from healthy individuals (Assinger et al., 2013). However, we did not observe an association between anti-CMV IgG titer and HERV-K(HM L-2) expression, suggesting that the reported effects of CMV on immunosenescence (reviewed in Söderberg-Naucler et al. 2016, Weltevrede et al. 2016) are not mediated via HERV-K(HM L-2) env expression.

Expression of HERV-K(HM L-2) env was used as an indicator of HERV-K(HM L-2) activation. The primers used in this study amplify the env sequence of 30 HERV-K(HM L-2) group members, yet there are over 90 HERVK(HM L-2) proviruses in the human genome (Subramanian et al., 2011). Although the env sequences of some of these proviruses are degraded, we cannot exclude that there might be additional HERV-K(HML-2) env expression not detected in our study. It remains to be understood whether the different members of the HERV-K(HML-2) group could have different effects on the immune system, depending on the proviral sequence or the location of the provirus in the genome. In addition, it is still not well understood how the amount of HERV-K(HM L-2) env mRNA corresponds to the amount of env protein.

\section{Author contributions}

All authors contributed to the recruitment of the cohort and sample collection. SM and TN performed the laboratory analyses. SM and M H wrote the manuscript. All authors approved the final manuscript.

\section{Competing financial interests}

The authors declare no competing financial interests.

\section{Funding}

This study was supported by grants to M H from the competitive Research Fund of Pirkanmaa Hospita District and from the Tampere Tuberculosis Foundation.

\section{Acknowledgements}

The authors would like to thank Sinikka Repo-Koskinen for technical assistance and IIkka Junttila and Laura Kummola for performing the FACS analysis. 


\section{References}

Assinger A, Yaiw KC, Göttesdorfer I, Leib-Mösch C, Söderberg-Nauclér C. 2013. Human cytomegalovirus (HCMV) induces human endogenous retrovirus (HERV) transcription. Retrovirology. 10:132.

Balestrieri E, Pica F, Matteucci C, Zenobi R, Sorrentino R, Argaw-Denboba A, Cipriani C, Bucci I, SinibaldiVallebona P. 2015. Transcriptional activity of human endogenous retroviruses in human peripheral blood mononuclear cells. Biomed Res Int. 2015:164529.

Beer L, M litz V, Gschwandtner M , Berger T, Narzt MS, Gruber F, Brunner PM Tschachler E, Mildner M . 2015. Bioinformatics approach for choosing the correct reference genes when studying gene expression in human keratinocytes. Exp Dermatol. 24(10):742-7.

Bhardwaj N, Maldarelli F, M ellors J, Coffin JM. 2014. HIV-1 infection leads to increased transcription of human endogenous retrovirus HERV-K (HM L-2) proviruses in vivo but not to increased virion production. J Virol. 88(19):11108-20.

Di Benedetto S, Derhovanessian E, Steinhagen-Thiessen E, Goldeck D, M üller L, Pawelec G. 2015. Impact of age, sex and CMV-infection on peripheral T cell phenotypes: results from the Berlin BASE-II Study. Biogerontology. 16(5):631-43.

Franceschi C, Campisi J. 2014. Chronic inflammation (inflammaging) and its potential contribution to ageassociated diseases. J Gerontol A Biol Sci Med suppl 1, 4-9.

Freimanis G, Hooley P, Ejtehadi HD, Ali HA, Veitch A, Rylance PB, Alawi A, Axford J, Nevill A, M urray PG, Nelson PN. 2010. A role for human endogenous retrovirus-K (HML-2) in rheumatoid arthritis: investigating mechanisms of pathogenesis. Clin Exp Immunol. 160(3):340-7.

Goebeler S, Jylhä M, Hervonen A. 2003. Medical history, cognitive status and mobility at the age of 90. A population-based study in Tampere, Finland. Aging Clin Exp Res 15, 154-161. 
Goronzy JJ, Weyand CM. 2013. Understanding immune senescence to improve vaccine responses. Nat Immunol 14, 428-436.

Hurme M, Paavilainen P, Pertovaara M, Jylhä M, Karhunen PJ, Hervonen A. Lehtimäki T. 2005. IgA levels are predictors of mortality in Finnish nonagenarians. Mech Ageing Dev 126, 829-831.

Kuparinen T, M arttila S, JylhäväJ, Tserel L, Peterson P, Jylhä M , Hervonen A, Hurme M . 2013. Cytomegalovirus (CMV) - dependent and -independent changes in the aging of the human immune system: A transcriptomic analysis. Exp Gerontol 48, 305-312.

Lang PO, Govind S, Aspinall R. 2013. Reversing T cell immunosenescence: why, who and how. AGE 35,609620.

M agiorkinis G, Belshaw R, Katzourakis A. 2013. 'There and back again': revisiting the pathophysiological roles of human endogenous retroviruses in the postgenomic era. Phil Trans R Soc B 368, 20120504.

M orandi E, Tanasescu R, Tarlinton RE, Constantinescu CS, Zhang W, Tench C, Gran B. 2017. The association between human endogenous retroviruses and multiple sclerosis: A systematic review and meta-analysis. PLoS One 12(2):e0172415.

Subramanian RP, Wildschutte JH, Russo C, Coffin JM. 2011. Identification, characterization, and comparative genomic distribution of the HERV-K (HML-2) group of human endogenous retroviruses. Retrovirology. 8:90.

Suntsova M, Garazha A, Ivanova A, Kaminsky D, Zhavoronkov A, Buzdin A. 2015. Molecular functions of human endogenous retroviruses in health and disease. Cell M ol Life Sci. 72(19):3653-75.

Söderberg-Naucler C, Fornara O, Rahbar A. 2016. Cytomegalovirus-driven immunosenescence - An immune phenotype with or without clinical impact? M ech Ageing Dev 158, 3-13 
Volkman HE, Stetson DB. 2014. The enemy within: endogenous retroelements and autoimmune diseases. Nat Immunol 15, 415-422.

Weltevrede E, Eilers R, de M elker HE, van Baarle D. 2016. Cytomegalovirus persistence in people aged fifty and older: A systematic review. Exp Gerontol 77, 87-95.

Zeng M. Hu Z, Shi X, Li X, Zhan X, Li X-D, Wang J, Choi JH, Wang K, Purrington T, Tang M. Fina M, DeBerardinis RJ, Moresco EY, Pedersen G, M clnerney GM, Karlsson Hedestam GB, Chen ZI, Beutler B. 2014. MAVS, cGAS, and endogenous retrovituses in T-independent B cell responses. Science 346, 1486-1492. 
Table 1. M edian and interquartile range of HERV-K(HM L-2) env expression and cell type proportion of PBM Cs from nonagenarians ( $n=61$ ) and young controls $(n=37)$. HERV-K(HM L-2) envexpression is presented as $\Delta \mathrm{Ct}$ value and cell type distributions are presented as percentages.

\begin{tabular}{|c|c|c|c|}
\hline & Nonagenarians & Young controls & $\begin{array}{l}\text { Mann-Whitney U- } \\
\text { test } p \text {-value }\end{array}$ \\
\hline HERV-K(HM L-2) env & $2.88(2.64-3.19)$ & $2.39(2.18-2.69)$ & $\varangle .001$ \\
\hline $\mathrm{CD} 14 t^{\mathrm{a}}$ & $9.28(2.51-14.85)$ & $5.03(1.82-10.50)$ & 0.031 \\
\hline $\mathrm{CD} 19+^{\mathrm{a}}$ & $2.36(1.56-4.51)$ & $6.18(4.52-8.90)$ & $\varangle 0.001$ \\
\hline $\mathrm{CD} 3 t^{\mathrm{a}}$ & $60.90(50.75-67.70)$ & $65.40(58.10-69.10)$ & 0.087 \\
\hline $\mathrm{CD} 4 t^{\mathrm{b}}$ & $53.50(46.20-68.25)$ & $57.60(53.55-62.45)$ & 0.695 \\
\hline $\mathrm{CD} 8+^{\mathrm{b}}$ & $29.70(21.45-43.65)$ & $30.30(26.75-35.45)$ & 0.725 \\
\hline $\mathrm{CD} 4+\mathrm{CD} 28-^{\mathrm{c}}$ & $17.20(7.10-33.20)$ & $1.50(0.95-4.25)$ & $\varangle 0.001$ \\
\hline 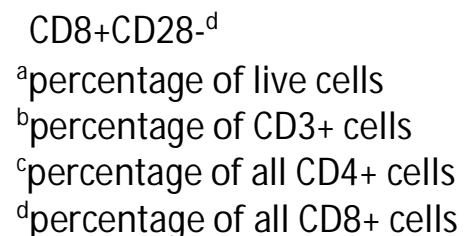 & $75.00(62.20-82.15)$ & $32.00(21.65-41.60)$ & $\varangle 0.001$ \\
\hline
\end{tabular}

Table 2. Correlation between the HERV-K(HM L-2) envexpression and proportions of different immune system cell types. As a smaller $\Delta \mathrm{Ct}$ value represents higher expression level, the negative correlation between the proportion of CD19+cells and HERV-K(HM L-2) env expression indicates that higher proportion of CD19+B cells is associated with higher level of HERV-K(HM L-2) env expression.

\begin{tabular}{|c|c|c|c|c|}
\hline & \multicolumn{2}{|c|}{ Nonagenarians } & \multicolumn{2}{|c|}{ Young controls } \\
\hline & Spearman's $\rho$ & $p$-value & Spearman's $\rho$ & $p$-value \\
\hline $\mathrm{CD} 14 t^{\mathrm{a}}$ & 0.156 & 0.229 & 0.067 & 0.692 \\
\hline $\mathrm{CD} 19 t^{\mathrm{a}}$ & -0.334 & 0.009 & -0.188 & 0.264 \\
\hline $\mathrm{CD} 3 t^{\mathrm{a}}$ & 0.036 & 0.782 & -0.265 & 0.113 \\
\hline $\mathrm{CD} 4 t^{\mathrm{b}}$ & -0.022 & 0.866 & -0.079 & 0.642 \\
\hline $\mathrm{CD} 8+^{\mathrm{b}}$ & -0.025 & 0.848 & -0.085 & 0.618 \\
\hline $\mathrm{CD} 4+\mathrm{CD} 28-^{\mathrm{C}}$ & 0.209 & 0.106 & 0.088 & 0.605 \\
\hline 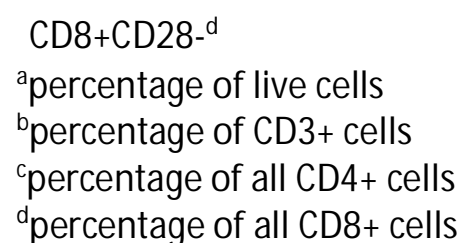 & 0.072 & 0.581 & 0.188 & 0.265 \\
\hline
\end{tabular}

